Pesq. Vet. Bras. 38(1):129-132, janeiro 2018

DOI: $10.1590 / \mathrm{S} 0100-736 \mathrm{X} 2018000100020$

\title{
Quantificação da IL-10 e do INF- $\gamma$ em cães com ou sem sinais clínicos de infecção com Leishmania (Leishmania) chagasi ${ }^{1}$
}

\author{
Carla J.R.M. do Rosário ${ }^{2 *}$, Marina F. Dominici ${ }^{3}$, Maria do Socorro C.O. Braga ${ }^{4}$, Cristian \\ A.A. Lima ${ }^{3}$, José G. Pereira ${ }^{4}$ e Ferdinan A. Melo ${ }^{4}$
}

\begin{abstract}
Rosário C.J.R.M., Dominici M.F., Braga M.S.C.B., Lima C.A.A., Pereira J.G. \& Melo F.A. 2018. [Quantification of IL-10 and IFN- $\gamma$ in dogs with or without clinical signs of Leishmania (Leishmania) chagasi infection.] Quantificação da IL-10 e do INF- $\gamma$ em cães com ou sem sinais clínicos de infecção com Leishmania (Leishmania) chagasi. Pesquisa Veterinária Brasileira 38(1):129-132. Departamento de Patologia, Universidade Estadual do Maranhão, Cidade Universitária Paulo VI s/n, Tirirical, São Luís, MA 65055-000, Brazil. E-mail: carlajanaina_rm@hotmail.com

Leishmaniasis comprise a complex of diseases caused by intracellular mandatory parasites belonging to the genus Leishmania. Considered as an important public health problem, and domestic dogs are primarily responsible for maintaining the epidemiological chain of the disease, it is estimated that more than the half of the dogs infected do not show clinical signs of the disease. The profile of IL-10 and IFN- $\gamma$ dogs naturally infected with Leishmania (Leishmania) chagasi in São Luís/MA was evaluated. Blood samples were collected from 50 animals, 20 from positive and symptomatic dogs for leishmaniasis canine (CVL), 20 from positive asymptomatic animals and 10 negative. Samples were analyzed by immunochromatographic test Dual Path Platform (DPP/Biomanguinhos ${ }^{\circledR}$ ) and by indirect ELISA (EIE/Biomanguinhos ${ }^{\circledR}$ ) for detection of anti-Leishmania antibodies. After the confirmation of the tests, the capture ELISA was performed for quantification of IL-10 and IFN- $\gamma$ cytokines through the Milliplex MAP kit. There was a statistical difference between the groups, observing an increase of IL-10 in blood of symptomatic dogs for CVL, compared to the group of asymptomatic animals, suggesting that animals with this expression of IL-10 may be associated with susceptibility to disease. As well as the increase in IFN- $\gamma$ levels in asymptomatic dogs, compared to the symptomatic dog group, could be related to chronicity of the disease.
\end{abstract}

INDEX TERMS: IL-10, IFN- $\gamma$, dogs, Leishmania chagasi, leishmaniasis, clinical signs, immunology.

RESUMO.- As leishmanioses compreendem um complexo de doenças causadas por parasitos intracelulares obrigatórios pertencentes ao gênero Leishmania. Consideradas como importante problema de saúde pública, sendo os cães

\footnotetext{
${ }^{1}$ Recebido em 1 de novembro de 2016.

Aceito para publicação em 11 de abril de 2017.

Realizado com apoio da Fundação de Amparo à Pesquisa e Desenvolvimento Científico e Tecnológico do Maranhão (Fapema).

${ }^{2}$ Doutoranda pela Rede de Biodiversidade e Biotecnologia da Amazônia Legal (Bionorte), Universidade Estadual do Maranhão (UEMA), Cidade Universitária Paulo VI s/n, Tirirical, São Luís, MA 65055-000, Brasil. Pesquisa de mestrado financiada pela CAPES. *Autor para correspondência: carlajanaina_rm@hotmail.com

${ }^{3}$ Graduado(a) em Medicina Veterinária, UEMA, Cidade Universitária Paulo VI s/n, Tirirical, São Luís, MA 65055-000, Brasil.

${ }^{4}$ Docente do Departamento de Patologia, UEMA, Cidade Universitária Paulo VI s/n, Tirirical, São Luís, MA 65055-000, Brasil.
}

domésticos os principais responsáveis pela manutenção da cadeia epidemiológica da doença, estima-se que mais da metade dos cães infectados não manifestam sinais clínicos da enfermidade. Avaliou-se o perfil de IL-10 e INF- $\gamma$ de cães naturalmente infectados com Leishmania (Leishmania) chagasi no município de São Luís-MA. Foram coletadas 50 amostras, sendo 20 de animais positivos e sintomáticos para Leishmaniose Visceral Canina (LVC), 20 de animais positivos e assintomáticos e 10 de animais sabidamente negativos para a LVC. As amostras foram analisadas pelo teste imunocromatográfico rápido Dual Path Platform (DPP/ Biomanguinhos $^{\circledR}$ ) e pelo ELISA (EIE/Biomanguinhos ${ }^{\circledR}$ ) indireto para detecção de anticorpos anti-Leishmania. Após as confirmações dos testes, foi realizado o ELISA de captura para quantificação das citocinas IL-10 e INF- $\gamma$ através do kit Milliplex MAP. Houve diferença estatística entre os gru- 
pos, observando um aumento de IL-10 em soros de cães sintomáticos para LVC, comparado com o grupo de animais assintomáticos, sugerindo que animais com essa expressão de IL-10 podem estar associados à susceptibilidade a doença. Assim como o aumento dos níveis de INF- $\gamma$ observados em cães assintomáticos, comparado com o grupo de cães sintomáticos, poderiam estar relacionados à cronicidade da doença.

TERMOS DE INDEXAÇÃO: IL-10, INF- $\gamma$, cães, Leishmania chagasi, leishmaniose, sinais clínicos, imunologia.

\section{INTRODUÇÃO}

As leishmanioses compreendem um grupo de doenças crônicas transmitidas por parasitas intracelulares do gênero Leishmania. Os cães domésticos são os principais responsáveis pela manutenção da cadeia epidemiológica da doença, pois vivem diretamente com o homem. Nesse contexto, a leishmaniose vem sendo considerada como importante problema de saúde pública (WHO 2013).

As diferentes manifestações clínicas estão relacionadas à espécie de Leishmania e a resposta imune do hospedeiro, podendo variar desde animais assintomáticos a animais sintomáticos apresentando diversas formas clínicas, como febre, perda de peso, esplenomegalia, hepatomegalia, anemia e úlceras, este último relacionado à leishmaniose cutânea (Handman \& Bullen 2002).

A infecção por Leishmania pode desencadear dois tipos de imunidade adaptativa associada à atividade dos linfócitos: a resposta humoral (anticorpos), associada à susceptibilidade à doença, e a resposta celular que preferencialmente promove a resistência à infecção (Rosa \& Machado 2011).

Em cães assintomáticos, a infecção por Leishmania (Leishmania) chagasi normalmente promove a doença sistêmica crônica, associada a uma resposta proliferativa específica, $m$ produção de INF- $\gamma$, IL- 2 e TNF- $\beta$ e baixos títulos de anticorpos anti-Leishmania. Já os cães sintomáticos apresentam depressão das funções das células T com produção de IL-10 e TGF- $\beta$ e altos níveis de anticorpos específicos (Corrêa et al. 2007).

O INF- $\gamma$ aumenta a expressão da enzima óxido nítrico sintase induzida (iNOS) nos macrófagos, gerando espécies reativas de nitrogênio e oxigênio, tais como o superóxido e o óxido nítrico, eficazes para a eliminação da Leishmania em ambiente intracelular (Goriely et al. 2009).

A IL-12 é a primeira citocina liberada em um tecido infectado e é essencial para a iniciação e polarização da resposta imune e também para a inibição da apoptose das células Th1. É produzida por células dendríticas e por macrófagos, desempenhando um papel importante na fase inicial da inflamação, porém por um curto período de tempo (Baneth 2006).

A IL-10 é capaz de desativar os macrófagos, inibindo suas funções leishmanicidas; suprimindo a apresentação de antígenos e a maturação das células dendríticas; além de suprimir a produção de INF- $\gamma$ pelas células T e NK; regulando negativamente a IL-12 e promovendo a sobrevida das células B, as quais também são fonte de IL-10. As células B, por sua vez, levam à produção de anticorpos e a formação de imunocomplexos, que acarretam em dano tecidual (Al- ves et al. 2009). Além da IL-10 alguns parasitas induzem a produção de TGF- $\beta$ que também exibe propriedades anti-inflamatórias (Gantt et al. 2003).

Alguns estudos relatam que a IL-10 não tem efeito inibitório direto sobre INF- $\gamma$ e IL-12, e que esta observação talvez não tenha relação com a imunossupressão da resposta mediada por células nestes animais ou com a doença severa associada à expressão da IL-10, pois alguns animais não conseguiram controlar a infecção (Quinnell et al. 2001, Santos-Gomes et al. 2002).

É importante ressaltar que a saliva do vetor tem função imunomodulatória (Paranhos et al. 1993).

Desta forma torna-se importante a realização do estudo da expressão de IL-10 e INF- $\gamma$, na infecção natural por Leishmania (Leishmania) chagasi com e sem manifestações clínicas no município de São Luís-MA.

\section{MATERIAL E MÉTODOS}

O projeto foi aprovado pelo Comitê de Ética em Experimentação Animal da Universidade Estadual do Maranhão (CEEA/UEMA), processo № 020/2014.

Amostras. Cinquenta cães independentes do sexo, raça e idade, foram selecionados aleatoriamente, dos atendimentos do Hospital Veterinário Universitário da Universidade Estadual do Maranhão (UEMA). Os cães foram submetidos ao exame clínico detalhado, bem como a realização de testes parasitológicos de fezes e pesquisas de hemoparasitas, os dados foram anotados em fichas clínicas individuais. Aqueles cães com infecção por Leishmania chagasi comprovada pelo teste rápido (DPP/Biomanguinhos $^{\circledR}$ ), e pelo teste sorológico (EIE-LVC/Biomanguinhos ${ }^{\circledR}$ ), e livres de outras doenças foram divididos em grupos com formas polares da doença: um grupo com vinte animais assintomáticos e outro com vinte animais com sinais bem definidos para LVC e um terceiro composto por 10 animais livres de infecção por L. chagasi.

Amostras de sangue foram obtidas através da venopunção da veia cefálica ou jugular dos animais e acondicionadas em tubos de ensaio. Logo após, o soro foi acondicionado em microtubo de polietileno e armazenado a $-20^{\circ} \mathrm{C}$ até a realização dos testes e avaliação do perfil de IL-10 e INF- $\gamma$.

Sorologia para leishmaniose. 0 teste de ELISA (EIE-LVC/ Biomanguinhos ${ }^{\circledR}$ ) indireto foi realizado com o objetivo de confirmar os animais positivos e negativos no DPP-Biomanguinhos ${ }^{\circledR}$, juntamente com a sintomatologia clínica dos cães, por serem oriundos de uma área endêmica e com um alto índice de transmissão. Identificando os que apresentavam título de anticorpos para Leishmania (Leishmania) chagasi. Os cães que apresentaram títulos baixos associados à clínica e aos demais índices, foram classificados em animais livres da infecção por Leishmania (Leishmania) chagasi e utilizados no grupo controle. 0 ponto de corte considerado para o ensaio foi de 0,375 determinado pela média da densidade óptica dos orifícios do controle negativo multiplicado por dois. As microplacas de polietileno de 96 poços (EIE-LVC/Biomanguinhos ${ }^{\circledR}$ ) foram previamente sensibilizadas com Leishmania major. Foram diluídos em tubos, $5 \mu \mathrm{L}$ dos controles e das amostras de soro homogeneizadas, em $500 \mu \mathrm{L}$ do diluente (1:100) disponibilizado no kit. Logo em seguida, na placa sensibilizada foram distribuídos $100 \mu \mathrm{L}$ dos controles e das amostras já diluídas. A placa foi levada à estufa a $37^{\circ} \mathrm{C}$ por 30 minutos. Logo após, o conteúdo da placa foi lavado $6 \mathrm{x}$ em seguida, com $200 \mu \mathrm{L} /$ orifício, aguardando 30 segundos entre cada lavagem. Foram homogeneizados e distribuídos $100 \mu \mathrm{L}$ da diluição do conjugado em cada poço da placa e incubada novamente a $37^{\circ} \mathrm{C}$ por $30 \mathrm{~min}$. Lavados novamente em solução PBS para então serem distribuídos 
$100 \mu \mathrm{L}$ do substrato em todos os orifícios. Deixado à temperatura ambiente e ao abrigo da luz, durante 30 minutos. Em seguida, foram adicionados $50 \mu \mathrm{L}$ de ácido sulfúrico $2 \mathrm{M}$ em todos os orifícios para bloquear a reação. A leitura foi realizada em espectrofotômetro para microplacas de 96 poços utilizando-se filtro para comprimento de onda de $450 \mathrm{~nm}$.

Determinação das citocinas. As citocinas (IL-10 e INF- $\gamma$ ) presentes nas amostras de plasma foram quantificadas utilizando uma plataforma multiplex customizada (MILLIPLEX MAP magnetic bead-based multi-analyte panels, Merck Millipore $\left.{ }^{\circledR}\right)$ e analisada em leitor de placas próprio (Luminex Multiplexing Instrument - EMD Millipore, Luminex Corporation, Austin, TX, USA). Conforme a metodologia do fabricante, as amostras foram adicionadas às placas específicas para o ensaio e diluídas em tampão fornecido pelo kit e, após a incubação com microesferas magnéticas fluorescentes recobertas por anticorpos de captura específicos para as citocinas listadas, realizou-se a leitura das amostras no equipamento. Os dados adquiridos foram analisados através do software Milliplex Analyst v3.5 (Millipore; VigeneTech Inc., Boston, EUA) utilizando uma curva logística de três parâmetros.

Análise estatística. A realização dos testes estatísticos foi com o apoio instrumental do software GraphPad Prism 5.0 (Prism Software, Irvine, CA, USA). A normalidade dos dados foi demonstrada pelo teste $\mathrm{T}$ de Student. Os testes de análise de variância (ANOVA) seguidos pelo teste de comparações múltiplas de Tukey foram empregados para avaliação entre os grupos do perfil de citocinas. Os dados obtidos foram considerados estatisticamente significativos quando o valor de $P<0,05$.

\section{RESULTADOS E DISCUSSÃO}

\section{Determinação da IL-10}

A IL-10 pode ter suprimido a resposta imunológica protetora em nosso estudo levando à progressão da doença com o aumento da expressão do parasito na célula, como encontrado por Iniesta et al. (2002), Bogdan et al. (1991), Karp et al. (1993), Wilson et al. (1998) e Bacellar et al. (2002).

Níveis superiores de IL-10 foram observados em cães com sintomatologia clínica para LVC, em relação ao grupo dos animais assintomáticos (Fig.1), essa relação indica que os macrófagos e as células T, dos cães infectados, estão produzindo IL-10 em resposta ao antígeno de Leishmania e tal produção talvez seja importante para que o parasito persista nas células do hospedeiro. Isso ocorre porque a IL-

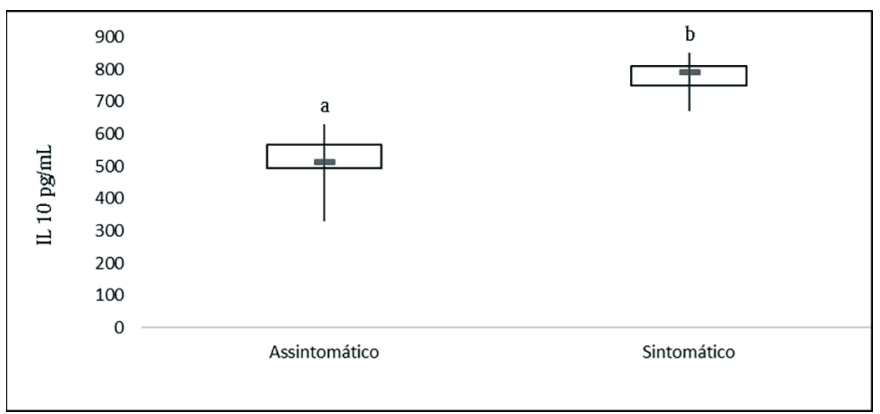

Fig.1. Quantificação da IL-10 no soro sanguíneo. Média e desvio-padrão do grupo de cães assintomáticos e sintomáticos, naturalmente infectados por Leishmania (Leishmania) chagasi, obtidos no ensaio de ELISA de captura. As letras a e b mostram diferenças significativas $(P<0,05)$ em comparação com os grupos assintomático e sintomático, respectivamente.
10 inibe a função das células apresentadoras de antígeno, pela diminuição da expressão de moléculas do complexo MHC de classe II e moléculas coestimulatórias, impossibilitando as funções de apresentação de antígenos por células dendríticas e macrófagos, já que a suscetibilidade a doença se deve a resposta regulatória Th2. A IL-10 também pode inibir a maturação e migração de células dendríticas, além de contribuir para a apoptose de células $\mathrm{T}$ e aumento da sobrevivência de células B, que podem ser fonte da geração de mais IL-10.

Além disso, ocorre também a inibição da produção de IL-12, o que pode resultar em inibição de uma resposta antígeno-específica do tipo Th1, necessária para o aumento da produção de INF- $\gamma$. Assim, o aumento da produção de anticorpos característico da LV pode contribuir para a patogênese da doença, causando danos teciduais pela deposição de imunocomplexos, que por sua vez podem estimular monócitos e macrófagos a produzirem mais IL-10 (Nylén \& Sacks 2007).

\section{Determinação do INF- $\gamma$}

Em nosso estudo níveis elevados de INF- $\gamma$ (Fig.2) no soro de cães assintomáticos em comparação com os níveis dos animais sintomáticos, naturalmente infectados por $L$. (L.) chagasi, mostram que a análise dessa relação poderia ser explicada pela estimulação a apresentação de antígenos pelo aumento da expressão de moléculas do MHC das classes I e II desencadeando uma resposta protetora à infecção, por meio da resposta Th1, corroborando com os achados de Santos-Gomes et al. (2002), Chamizo et al. (2005).

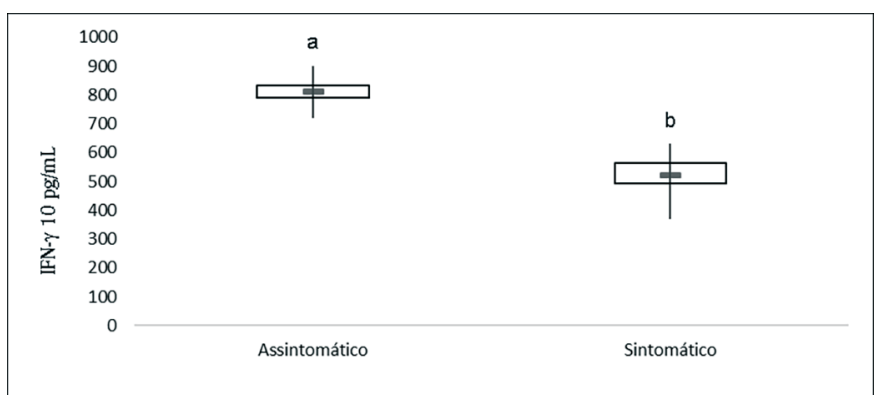

Fig.2. Quantificação do IFN- $\gamma$ no soro sanguíneo. Média e desvio-padrão do grupo de cães assintomáticos e sintomáticos, naturalmente infectados por Leishmania (Leishmania) chagasi, obtidos no ensaio de ELISA de captura. As letras a e b mostram diferenças significativas $(P<0,05)$ em comparação com os grupos assintomático e sintomático, respectivamente.

Contrariando os achados de um trabalho desenvolvido por Sanchez-Robert et al. (2008) que foi demonstrado um aumento da expressão de INF- $\gamma$ em CMSP nas formas clínicas mais graves da LVC, o que também aponta para o papel imunopatológico dessas citocinas nos processos relacionados a inflamação generalizada e visceral.

O INF- $\gamma$ é produzido por células T, dendríticas, macrófagos e células "natural killer" (NK) e tem a função de induzir a síntese de óxido nítrico, ativando funções microbicidas possuindo um espectro de ação muito amplo, sendo considerada uma das principais citocinas estimuladoras 
da imunidade contra infecções, atuando em ambas as respostas imunes, inata e adaptativa. É considerado o principal ativador de macrófagos, estimula a resposta imune por favorecer o crescimento de células Th1 em detrimento das Th2, e estimula a apresentação de antígenos pelo aumento da expressão de moléculas do MHC das classes I e II.

Dessa forma, os resultados provenientes da análise da expressão de INF- $\gamma$, possibilitam inferir que a expressão aumentada e simultânea desses mediadores deve estar intimamente envolvida com mecanismos imunoprotetores durante a LVC, visto que, observou-se aumento dessas citocinas no soro de animais portadores da forma clínica assintomática.

\section{CONCLUSÃO}

Os resultados obtidos sugerem que o aumento dos níveis de IL-10 no soro dos cães portadores dos sinais clínicos para LVC podem estar relacionados a susceptibilidade à doença, quando comparado com os níveis dos cães assintomáticos analisados. Da mesma maneira que o aumento dos níveis de INF- $\gamma$ no soro dos cães assintomáticos observados pode estar associado à cronicidade da infecção.

Agradecimentos.- À CAPES e à Fundação de Amparo a Pesquisa e Desenvolvimento Científico e Tecnológico do Maranhão (Fapema).

\section{REFERÊNCIAS}

Alves C.F., De Amorim I.F., Moura E.P., Ribeiro R.R., Michalick M.S., Kalapothakis E., Bruna-Romero O., Tafuri W.L., Teixeira M.M. \& Melo M.N. 2009. Expression of IFN gamma, TNF-alpha, IL-10 and TGF-beta in lymph nodes associates with parasite load and clinical form of disease in dogs naturally infected with Leishmania (Leishmania) chagasi. Vet. Immunol. Immunopathol. 128:349-358.

Bacellar O., Lessa H., Schriefer A., Machado P., Jesus A.R., Dutra W.O., Gollob K.J. \& Carvalho E.M. 2002. Up- regulation of Th1-type responses in mucosal Leishmaniasis patients. Infect. Immun. 70:6734-6740.

Baneth G. 2006. Leishmanasis, p.685-698. In: Greene C.E. (Ed.), Infectious Diseases of the Dog and Cat. 3rd ed. Saunders Elsevier, Canada.

Bogdan C., Vodovotz Y. \& Nathan C. 1991. Macrophage deactivation by interleukin 10. J. Exp. Med. 174:1549-1555.

Chamizo C., Moreno J. \& Alvar J. 2005. Semi-quantitative analysis of cytokine expression in asymptomatic canine leishmaniasis. Vet. Immunol. Immunopathol. 103:67-75.

Corrêa A.P.F.L., Dossi A.C.S., Vasconcelos R.O., Munari D.P. \& Lima V.M.F.
2007. Evaluation of transformation growth factor $\beta_{1}$, interleukin-10, and interferon- $\gamma$ in male symptomatic and asymptomatic dogs naturally infected by Leishmania (Leishmania) chagasi. Vet. Parasitology. 143:267-274.

Gantt K.R., Schultz-Cherry S., Rodriguez N., Jeronimo S.M., Nascimento E.T., Goldman T.L., Recker T.J., Miller M.A. \& Wilson M.E. 2003. Activation of TGF-beta by Leishmania chagasi: importance for parasite survival in macrophages. J. Immunol. 170:2613-2620.

Goriely S., Cavoy R. \& Goldman M. 2009. Interleukin-12 family members and type interferons in Th17-mediated inflammatory disorders. Allergy 64:702-709.

Handman E. \& Bullen D.V.R. 2002. Interaction of Leishmania with the host macrophage. Trends Parasitol. 18(8):417.

Iniesta V., Carcélen J., Molano I., Peixoto P.M.V., Redondo R., Parra P., Mangas M., Moroy I., Campo M.L., Nieto C.G. \& Corraliza I. 2002. Arginase I induction in macrophages, triggered by Th2-type cytokines, supports the growth of intracellular Leishmania parasites. Parasite Immunol. 24:113-118.

Karp C.L., El-Safi S.H., Wynn T.A., Satti M.M., Kordofani A.M., Hashim F.M., Hag-Ali M., Neva F.A., Nutman T.B. \& Sacks D.L. 1993. In vivo cytokine profiles in patients with kala-zar: marked elevation of both interleukin-10 and interferon-gamma. J. Clin. Invest. 91:1644-1648.

Nylén S. \& Sacks D. 2007. Interleukin-10 and the pathogenesis of human visceral leishmaniasis. Trends Immunol. 28:378-384.

Paranhos M., Dos-Santos W.C., Sherlock I., Oliveira G.G. \& Pontes-de-Carvalho L.C. 1993. Development of eosinophilia in dogs intradermically inoculated with sand fly saliva and Leishmania (Leishmania) chagasi stationary-phase promastigotes. Mem. Inst. Oswaldo Cruz. 88:249-251.

Quinnell R.J., Courtenay O., Shaw M.A., Day M.J., Garcez L.M., Dye C. \& Kaye P.M. 2001. Tissue cytokine responses in canine visceral leishmaniasis. J. Infect. Dis. 183:1421-1424.

Rosa M.E.A. \& Machado P.RL. 2011. Disseminated Leishmaniasis: clinical, immunological, and therapeutic aspects. Drug Dev. Res. 72:437-441.

Sanchez-Robert E., Altet L., Alberola J., Rodriguez-Cortes A., Ojeda A., López-Fuertes L., Timón M., Sanchez A. \& Francino O. 2008. Longitudinal analysis of cytokine gene expression and parasite load in PBMC in Leishmania infantum experimentally infected dogs. Vet. Immunol. Immunopathol. 15:168-175.

Santos-Gomes G.M., Rosa R., Leandro C., Cortes S., Romão P. \& Silveira H. 2002. Cytokine expression during the outcome of canine experimental infection by Leishmania infantum. Vet. Immunol. Immunopathol. 88:2130 .

Wilson M.E., Young B.M., Davidson B.L., McDevitt H.O \& Duff G.W. 1998. The importance of transforming growth factor $\mathrm{b}$ in murine visceral leishmaniasis. J. Immunol. 161:6148-6155.

WHO 2013. Leishmaniasis. World Health Organization, Genebra. Disponível em <http://www.who.int/leishmaniasis/en/index.html> Acesso em 6 abr. 2014. 